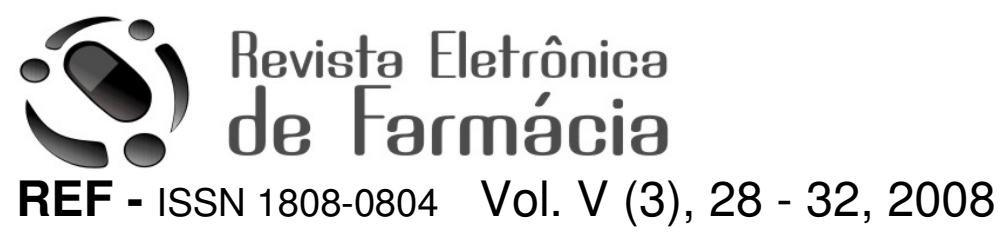

\title{
INVESTIGAÇÃO SOBRE O USO RACIONAL DE MEDICAMENTTOS NO MUNICÍPIO DE QUIXADÁ
}

\section{Research on the rational use of drugs in municipality of quixadá}

\author{
Rachel Ferreira da Silva ${ }^{1}$, Rivelilson Mendes de Freitas ${ }^{2 *}$ \\ ${ }^{[1]}$ Acadêmica do Curso de Farmácia da Faculdade Católica Rainha do Sertão. \\ ${ }^{[2]}$ Professor Adjunto. Setor de Fisiologia e Farmacologia da Universidade Federal do Piauí. - UFPI. Rua Cícero \\ Eduardo, s/n, Junco, Picos, 64600-000, Piauí.
}

*Autor para correspondência e-mail: rivelilson@ufpi.br

Recebido em 05/10/2008 - Aceito em 16/11/2008

\begin{abstract}
RESUMO: No presente estudo avaliou-se o uso irracional de medicamentos pela população usuária dos serviços farmacêuticos de uma farmácia comunitária no município de Quixadá, em particular observou-se o perfil farmacoepidemiológico, os problemas de saúde, a forma de aquisição, o armazenamento, as hipóteses diagnósticas que induzem a automedicação, a adesão ao tratamento e as reações adversas aos medicamentos (RAMs). Todos os pacientes atendidos eram adultos e à faixa etária mais prevalente foi a de 35-50 anos, sendo $38 \%$ do sexo masculino e $62 \%$ do sexo feminino. A maioria (65\%) estudou somente até o ensino fundamental, eram casados ou tinham uma relação estável. A principal reação adversa observada foi à diarréia (35\%), enquanto que esta patologia (30\%) foi a maior responsável pela automedicação e o tabagismo o principal problema relacionado à saúde $(55 \%)$. E entre os problemas relacionados aos medicamentos (PRM) o mais prevalente foi o de não adesão ao tratamento farmacológico (72\%), foi visto também que a maioria dos medicamentos adquiridos são armazenados de forma incorreta (47\%). Os dados obtidos indicam a necessidade de implantação de um serviço de atenção farmacêutica, para orientação dos usuários e consequentemente melhora na aderência ao esquema terapêutico, e uma redução na prática da automedicação.
\end{abstract}

PALAVRA-CHAVE: Automedicação, medicamentos, atenção farmacêutica, uso irracional dos medicamentos.

\section{INTRODUÇÃO}

A Organização Mundial da Saúde (OMS) define a utilização de medicamentos como a comercialização, distribuição, prescrição, dispensação e uso de medicamentos na sociedade, e com especial destaque sobre as conseqüências médicas, sociais e econômicas (OMS, 1997). A informação sobre medicamentos atual, imparcial e imune de pressões políticas e econômicas pode subdisiar o uso racional dos fármacos como agente de promoção, de proteção e/ou de recuperação da saúde. Os fármacos de um modo geral ocupam um importante papel no sistema de saúde, uma vez que reduz a taxa de mortalidade, cura e/ou suprime os sinais e sintomas de inúmeras patologias (OMS, 1993; NOELLE, 2002). O amplo emprego dos medicamentos, os altos custos que estes representam a assistência à saúde, a elevada incidência de morbidade e mortalidade atribuída a estes, pode ser prevenida ou reduzida por uma assistência farmacêutica de qualidade, tornando o uso racional dos medicamentos um dos grandes desafios para a implementação desta no contexto da saúde pública (AFONSO \& PUERTA, 1991; WINTERSTEIN et al., 2002).

A automedicação pode induzir a morbidade e mortalidade relacionada aos medicamentos. Chaves, (1999) estudando o uso racional de medicamentos verificou que a utilização de forma correta e racional destes pode otimizar o atendimento à saúde, promovendo, assim, uma conseqüente redução dos gastos com saúde, uma menor incidência de morbidade e mortalidade relacionada aos medicamentos, uma diminuição no tempo de internação hospitalar e uma melhor qualidade de vida.

A redução da automedicação pode ser induzida através da aplicação da prática da atenção farmacêutica em diferentes setores da saúde. O objetivo dessa prática é prover de forma responsável a farmacoterapia visando alcançar resultados definidos que melhorem a qualidade de vida dos pacientes (FAUS, 2000). Esta prática pode, então, reduzir os problemas relacionados a medicamentos, tais como a automedicação, a 
Sílvia R. F. et al./Revista Eletrônica de Farmácia Vol 5(3), 28 - 32, 2008.

morbidade, a mortalidade, as reações adversas a medicamentos (RAMs), a ausência da adesão ao tratamento, entre outros.

A utilização de medicamentos é um processo complexo com múltiplos determinantes e envolve diferentes setores da saúde. A automedicação é uma prática difundida em vários países, inclusive no Brasil, sendo um dos principais agravantes da política de saúde e uma das principais preocupações da profissão farmacêutica. Easton e colaboradores (1998) investigando a automedicação verificaram que a mesma pode ser induzida pela má prescrição médica devido à dificuldade de acesso aos serviços de saúde, bem como pela ausência de orientação médica sobre posologia, duração do tratamento, reações adversas, interações medicamentosas (medicamentomedicamento e medicamento-alimento) e efeitos colaterais.

Atualmente, o grande avanço nas tecnologias, o aumento da disponibilidade de novos agentes terapêuticos mais eficazes, o elevado número de propagandas sobre medicamentos pelas grandes indústrias farmacêuticas nacionais e internacionais e, ainda devido à ausência de uma adequada política de assistência à saúde, ocorre à aquisição e utilização de forma abusiva de medicamentos sem nenhuma preocupação com a relação ao risco/benefício e/ou custo/efetividade da terapia medicamentosa sem prescrição médica (CAVALLINI \& BISSON, 2002).

\section{OBJETIVOS}

Como a maioria da população brasileira utiliza uma grande variedade de medicamentos sem prescrição médica devido às doenças associadas, este trabalho propôs delinear o perfil sócio-econômico dos usuários dos serviços de uma Farmácia Comunitária no município de Quixadá, investigar os principais medicamentos utilizados por estes e, ainda avaliar os principais problemas relacionados com a automedicação.

\section{MATERIAL E MÉTODOS}

As entrevistas seguiram a metodologia proposta por Cipolle, et al., 2002. Os resultados foram obtidos por meio de um estudo prospectivo observacional das entrevistas realizadas na Farmácia Comunitária no município de Quixadá no período de julho de 2006 a julho de 2007.

\section{Coleta dos dados}

A coleta dos dados dos pacientes foi realizada pelos acadêmicos de Farmácia da Faculdade Católica Rainha do Sertão, treinados sob supervisão, sendo realizada por meio de entrevista com o usuário para se obter as seguintes informações: a idade, o sexo, o estado civil, a escolaridade, os problemas de saúde, a forma de aquisição, o armazenamento, as hipóteses diagnósticas que induzem a automedicação, a adesão ao tratamento e as reações adversas aos medicamentos (RAMs). Não houve identificação nominal, nem risco moral para os pacientes, por se tratar apenas de dados estatísticos.

\section{Análise dos dados}

As informações coletadas foram lançadas em sistema informatizado Excel ${ }^{\circledR}$, versão 2003, para análise dos resultados em percentagem.

\section{RESULTADOS E DISCUSSÃO}

Foi acompanhado um total de 400 pacientes, destes 180 (45\%) apresentaram faixa etária entre 35 e 50 anos, seguidos de 100 usuários (25\%) com menos de 20 anos, de 81 usuários com idade entre 20 a 30 anos e 39 usuários (9\%) com idade superior a 50 anos. As entrevistas indicaram a prevalência do sexo feminino 248 $(62,0 \%)$. Dentre as opções para o estado civil, $240(60 \%)$ deles eram casados, $116(29 \%)$ solteiros e $44(11 \%)$ viúvos. Com relação à escolaridade $260(65 \%)$ tinham o ensino fundamental completo, 96 (23\%) eram analfabetos e 44 (12\%) completaram o ensino médio.

Os problemas relacionados à saúde mais comuns detectados foram: o tabagismo $220(55 \%)$, o etilismo $128(32 \%)$, a insônia $36(9 \%)$ e a dependência à cafeína 16 (4\%).

Dos pacientes entrevistas 224 (56\%) são responsáveis pela aquisição sem prescrição médica de medicamentos para o próprio consumo, enquanto que $116(29 \%)$ e $60(15 \%)$ destes foram os parentes e os amigos, respectivamente, que adquirem os medicamentos para outros usuários.

Quando questionados sobre a maneira do armazenamento dos medicamentos, 188 (47\%) dos entrevistados responderam que armazenam os medicamentos no armário do banheiro, 116 (29\%) dentro da geladeira, 60 (15\%) em cima da geladeira, 20 (5\%) na cabeceira da cama e $16(4 \%)$ em outros locais.

Foi identificado durante a pesquisa que as principais hipóteses diagnósticas que induzem ao consumo de medicamentos sem prescrição médica (automedicação) são 120 (30\%) diarréia, 92 (23\%) dor, 72 (18\%) infecção, 56 (14\%) gripe, 40 (10\%) febre e $20(5 \%)$ outros. 
Sílvia R. F. et al./Revista Eletrônica de Farmácia Vol 5(3), 28 - 32, 2008.

Quanto às repostas sobre a adesão ao tratamento foi verificado que $180(45 \%)$ usuários quando esquecem de tomar uma dose do medicamento, tomam dois comprimidos numa única vez, 116 (29\%) pulam a dose, 81 (21\%) pára de tomar a medicação e 23 (5\%) não responderam. 288 (72\%) usuários abandonam o tratamento quando ocorre à supressão dos sinais e sintomas da patologia e, apenas $72(18 \%)$ cumprem o tratamento completo. Dos entrevistados $40(10 \%)$ afirmaram que a adesão ou não ao tratamento ocorre de forma diferenciada, uma vez que depende da evolução dos sintomas da patologia.

As reações adversas aos medicamentos (RAMs) que apresentaram maior freqüência foi a diarréia 140 (35\%), cefaléia $116(29 \%)$, constipação $72(18 \%)$, fadiga $20(5 \%)$ e $52(13 \%)$ outros. Após o aparecimento das RAMs, $300(75 \%)$ usuários param o tratamento, $44(11 \%)$ suspendem por alguns dias e depois retoma o tratamento, $40(10 \%)$ deles diminuem a dose e somente 16 (4\%) usuários realizam o tratamento completo. De acordo com o levantamento bibliográfico realizado foi detectado que os pacientes procuram a farmácia comunitária devido à dificuldade de acessos aos serviços de saúde do Sistema Único de Saúde, como também em conseqüência de uma internação temporária que quando apresentam melhora, retornam as suas residências devido à alta hospitalar, mas que podem ainda requerer algum tipo de medicamento com ou sem prescrição médica (FONSECA et al., 2006).

A faixa etária de maior prevalência foi de 35-50 anos identificando esta faixa etária como um dos fatores de risco para o desenvolvimento de morbidades e mortalidades relacionadas a medicamentos (FAUCl, et al, 2002). O sexo feminino foi o predominante dentre os usuários entrevistados, o que pode estar correlacionado sexo e idade, uma vez que é nessa fase que ocorre a menopausa. Esta etapa caracteriza-se pela redução na produção de hormônios provenientes do colesterol, estando este não consumido, aumentando sua concentração sérica e 0 risco de aderir nos vasos sanguíneos e desencadear doenças cardiovasculares como trombose, infarto e também neurológicos como o acidente vascular cerebral (AVC) e isquemia ( $\mathrm{FAUCl}$, et al, 2002). A maioria dos usuários apresenta estado civil casado e ensino fundamental completo, esses dados corroboram com os apresentados pelo IBGE (2007) para o município de Quixadá e são semelhantes aos apresentados por outros municípios do Sertão Central do Nordeste brasileiro.

Foi observada uma correlação entre os problemas relacionados à saúde mais freqüentes entre pacientes e as hipóteses diagnósticas, pois alguns desses são fatores de risco para o desencadeamento das doenças apresentadas, como por exemplo, o tabagismo para acidente vascular cerebral e hipertensão arterial sistêmica, além do uso de contraceptivo associado ao tabagismo que também poder aumentar esse risco. Assim, como o etilismo pode causar hipertensão arterial sistêmica (FAUCl et al, 2002) e seu agravamento pelo tabagismo (KODA-KIMBLE, et al., 2001). Pode-se observar que os medicamentos mais prescritos de acordo com o levantamento estão envolvidos com maior prevalência de problemas relacionados com efetividade e segurança ressaltando a necessidade de adoção de parâmetros de monitorização desses aspectos na clínica baseada em evidências, a fim de previr complicações de interações medicamentos e reações adversas.

De acordo com os dados verificados sobre a automedicação foi observado que mais da metade dos entrevistados é responsável pela aquisição do seu próprio medicamento, fazendo uso dessa medicação de acordo com a melhor conveniência não havendo a preocupação com a conduta terapêutica correta, uma vez que, estes procuram a farmácia sem prescrição médica. O armazenamento das medicações é feito pela grande maioria dos usuários de forma incorreta, já que são armazenados em locais com umidade e temperatura inadequados que possibilitam o crescimento de microrganismos e a decomposição do princípio ativo dos medicamentos, que podem, assim, induzir outros problemas relacionados a medicamentos (PRM) como à falta de eficácia terapêutica e as RAMs.

A principal hipótese diagnóstica que induziu a prática incorreta do uso de medicamentos foi à algesia em geral. A adesão ao tratamento farmacológico, revelou sérias preocupações quanto à farmacoterapia, já que a grande maioria da população ao esquecer uma dose faz o uso do medicamento no dobro da dose prescrita ou não, como forma compensatória. Essa prática incorreta durante os tratamentos pode induzir ao aparecimento de outro PRM, como no caso à intoxicação.

A automedicação é uma prática bastante difundida não apenas no Brasil, mas também em outros países subdesenvolvidos e desenvolvidos. Em alguns países cujo sistema de saúde é pouco estruturado, a procura pelos serviços de farmácias comunitárias pode representar a primeira opção para resolver um problema de saúde, uma vez que a maior parte dos medicamentos adquiridos pela população é comercializada de forma livre sem prescrição médica (FREITAS et al., 2005). Vários medicamentos (analgésicos, antitérmicos, antiinflamatórios, etc) estão disponíveis em farmácias, drogarias ou supermercados e podem ser obtidos sem necessidade de prescrição médica (EDITORIAL, 2001). A assistência farmacêutica objetiva atender aos pacientes, prestando um atendimento qualificado, a fim de promover o uso racional, com eficácia e segurança, podendo, assim, ser evitado vários problemas relacionados a medicamentos (GOMES \& REIS, 2000).

A automedicação deve ser evitada, já que pode ocasionar uma série de PRMs, independente da classe sócio-econômica ou do grau de instrução da população em geral (STORPIRTIS, 1999). A automedicação é um dos grandes indutores de PRMs, que em geral pode resultar em agravamento do quadro clínico, tornando mais elevados os custos com o tratamento farmacológico e aumentando o tempo de internação hospitalar (ALVAREZ et al., 2001; ALBEROLA et al., 1991 e HOLLAND \& NIMMO, 1999). 


\section{CONCLUSÕES}

A realização da Atenção Farmacêutica em Farmácia Comunitária é de difícil implantação devido à reduzida inserção do profissional farmacêutico neste contexto de assistência à saúde. Apesar disso, é de suma importância para a racionalização da terapia farmacológica e melhor qualidade de vida da população, visando o uso racional de medicamentos pela população residente no município de Quixadá.

Alguns problemas relacionados ao medicamento podem ser detectados e resolvidos por meio da adoção de orientação quanto ao uso racional. O farmacêutico deve fornecer informações sobre a terapia medicamentosa prevenindo, detectando e corrigindo PRMs, tais como a não adesão ao tratamentos, a sobredose, as reações adversas, as interações medicamentosas, a falta de eficácia e as intoxicações. Desta forma, garante-se o sucesso da terapia farmacológica, melhorando a qualidade de vida do paciente através da redução de problemas relacionados à saúde, contribuindo dessa forma também para redução dos custos assistenciais.

\section{REFERÊNCIAS BIBLIOGRÁFICAS}

ALVAREZ, T.F., GONZALES, P.A., RIERA, T.E. - Pharmaceutical care in people who have acute coronory episodes. Rev. Esp. Salud Publica, v. 75, p. 375-388, 2001.

AFONSO, G.M.T., PUERTA, F.A.M.C. - Auto valoración social, económica y profesional del farmacéutico comunitario. Rev O.F.I.L, v. 5, p. 298-303, 1991.

ALBEROLA, G.E.C.; CRUZ, M.E.; CRUZ, T. - Farmacovigilancia el aténción primaria: experiencia en centro de salud. Rev O.F.I.L, v. 2, p. 85-88, 1991.

CAVALLINI, M.E.; BISSON, M.P. - Farmácia Hospitalar - Um enfoque em sistemas de saúde. Manole, $1^{a}$ ed, Barueri, São Paulo, 2002.

CHAVES, A. Estudios de utilización de medicamentos: conceptos e aplicaciones. In: BERMUDEZ, J.R.A. Medicamentos e a reforma do setor saúde. São Paulo: Hucitec/Sobravime, p. 101-124, 1999.

EASTON, K.L.; BARRY, T.P.; STARR, M.S. et al. - The incidence of drug related problems as a cause of hospital admission in children, M.J.A., v. 168, p. 356-359, 1998.

EDITORIAL. - Automedicação. Revista da Associação Médica Brasileira, v. 47, p. 1-4, 2001.

FAUCI, A.S. et al. Harrison - tratado de Medicina Interna, 15 ed. Madri: Mc Graw Hill, 2002.

FAUS, M.J. - Atención farmacéutica como-respuesta a una necesidad social. Ars Pharmaceutica, v. 41, p. 137$143,2000$.

FONSECA, C.A., OLIVEIRA, A.M., SOUSA, L.G., COSTA, L.F. - Atenção farmacêutica para portadores de cuidados especiais, Revista Eletrônica de Farmácia, v. 3, p. 19-21, 2006.

FREITAS, R.M.; BRAGA, D.S.; BORGES, K.D.M.; IODES, A.M.F. - Estudo do uso racional de medicamentos por usuários do Centro de Atenção Psicossocial, Revista Pharmacia Brasileira, v.17, p.74-77, 2005.

GOMES, M.J.V.M.; REIS, A.M.M. - Novas diretrizes para assistência farmacêutica hospitalar: atenção farmacêutica/farmácia clínica, Ciências farmacêuticas - uma abordagem em Farmácia Hospitalar. Atheneu, p. 521-525, 2000.

HOLLAND, R.W.; NIMMO, C.M. - Transitions part 1: Beyond pharmaceutical care. Am J Health Syst Pharm, v. 56, p. 1758-1764, 1999.

IVAMA, A.M. et al. Consenso brasileiro de atenção farmacêutica: proposta. Brasília: Organização Pan-Americana da Saúde, 2002.

KODA-KIMBLE, M.A., YOUNG, L.Y. Applied therapeutics - the clinical use of drugs. Philadelphia: Lippincott Willians \& Wilkins, 2001.

MOREAS, I.N. - Conforto da automedicação - importância e perigos. ROCA, v.1, p. 9-67, 2004. 
Sílvia R. F. et al./Revista Eletrônica de Farmácia Vol 5(3), 28 - 32, 2008.

NOELLE, L. - Bioethics and 21st century, view point of the jurist. Presse Med, v. 31, n. 12, p. 565-570, 2002.

ORGANIZAÇÃO MUNDIAL DE SAÚDE - OMS - El papel del farmaceutico en aténcion a la salud: declaración de Tokio, Genebra, 1993.

ORGANIZAÇÃO MUNDIAL DE SAÚDE - OMS - The state of world health: life expectancy, health expectancy, 1997.

STORPIRTIS, S. - Farmácia Clínica. Rev. Farm. Quím, v. 32, n. 1, p. 33-34, 1999.

WINTERSTEIN, A.G., SAUER, B.C., HEPLER, C.D. et al., - Preventable drug related hospital admission. Ann. Pharmacother., v. 36, p. 1238-1248, 2002. 